\title{
DEGREE OF SYMMETRY OF A HOMOTOPY REAL PROJECTIVE SPACE
}

\author{
BY \\ H. T. KU, L. N. MANN, J. L. SICKS AND J. C. SU( $\left.{ }^{(}\right)$
}

\begin{abstract}
The degree of symmetry $N(M)$ of a compact connected differentiable manifold $M$ is the maximum of the dimensions of the compact Lie groups which can act differentiably and effectively on it. It is well known that $N(M) \leqq \operatorname{dim} S O(m+1)$, for an $m$-dimensional manifold, and that equality holds only for the standard $m$ sphere and the standard real projective $m$-space. W. Y. Hsiang has shown that for a high dimensional exotic $m$-sphere $M, N(M)<m^{2} / 8+1<\left(\frac{1}{4}\right) \operatorname{dim} S O(m+1)$, and that $N(M)=m^{2} / 8+7 / 8$ for some exotic $m$-spheres. It is shown here that the same results are true for exotic real projective spaces.
\end{abstract}

0. Introduction. The degree of symmetry $N(M)$ of a compact connected differentiable $m$-manifold $M^{m}$ is the maximum of the dimensions of the compact Lie groups which can act effectively and differentiably on $M$. It is well known that

$$
N\left(M^{m}\right) \leqq m(m+1) / 2,
$$

and that if $N\left(M^{m}\right)=m(m+1) / 2$, then $M$ is diffeomorphic to the standard sphere $S^{m}$ or the standard real projective space $R P^{m}$ [3]. In [6] W. Y. Hsiang showed that if $\Sigma^{m}$ is an exotic sphere $(m \geqq 40)$, then

$$
N\left(\Sigma^{m}\right)<m^{2} / 8+1 .
$$

Hsiang's result is best possible in the sense that

$$
N\left(\Sigma_{0}^{8 k+1}\right)=m^{2} / 8+7 / 8
$$

where $\Sigma_{0}^{8 k+1}$ is the Kervaire sphere of dimension $m=8 k+1$.

In this paper we complement Hsiang's result by showing that if $M$ is a homotopy real projective $m$-space ( $m \geqq 72$ ), not diffeomorphic to $R P^{m}$, then

$$
N(M)<m^{2} / 8+1 .
$$

Again this bound is best possible, as we exhibit an exotic homotopy real projective $m$-space (whose universal covering space is actually $\Sigma_{0}^{8 k+1}, m=8 k+1$ ) with degree of symmetry $m^{2} / 8+7 / 8$.

Received by the editors April 23, 1970.

AMS 1969 subject classifications. Primary 2240, 5747; Secondary 5480.

Key words and phrases. Differentiable manifold, compact Lie group, degree of symmetry, exotic sphere, exotic real projective space.

(1) The second and fourth authors were supported in part by NSF 11662; the third author by NSF 13281 .

Copyright (C) 1971, American Mathematical Society 
Let us comment briefly on our approach. Suppose $M$ is a homotopy $R P^{m}$ with $N(M) \geqq m^{2} / 8+1$ and let $G$ be a compact connected Lie group of dimension $N(M)$ acting effectively and differentiably on $M$. Then the action of $G$ on $M$ can be lifted to an effective and differentiable action of a covering group $\tilde{G}$ of $G$ on the universal covering space $\tilde{M}$ of $M$ [9]. Now $\tilde{M}$ is a homotopy $m$-sphere and

$$
N(\tilde{M}) \geqq \operatorname{dim} \tilde{G}=\operatorname{dim} G=N(M) \geqq m^{2} / 8+1 .
$$

Hence by the previously mentioned result of W. Y. Hsiang, $\tilde{M}$ must be a standard sphere. However, although $M$ is covered by the standard sphere $S^{m}$ the deck involution on $S^{m}$ could conceivably be exotic, and hence $M$ might be an exotic homotopy $R P^{m}$. Our approach, then, is to establish a structure theorem directly for actions of compact Lie groups on homotopy $R P^{m}$ 's. We show that if $M^{m}$ is a homotopy $R P^{m}$ admitting an effective differentiable action of a compact connected Lie group with $S^{k}(k \neq 1,3)$ as principal orbit, then $M$ is diffeomorphic to $R P^{m}$. The proof of the structure theorem seems to hinge on the fact that the Whitehead group of the group $Z_{2}$ is trivial. Our structure theorem is analogous to one for homotopy spheres used by W. Y. Hsiang in [6] and with it we are able to establish our bound for the degree of symmetry of exotic $R P^{m}$ 's.

For the sake of clarity we collect some preliminaries in the next two sections.

1. Preliminaries. Let $G$ be a compact connected Lie group acting smoothly on a compact connected manifold $M$ such that the fixed-point set $F(G, M)$ is nonempty. Choose a base point $x_{0} \in F(G, M)$, let $\tilde{M}$ be the universal covering space of $M$ represented as homotopy classes of paths in $M$ starting at $x_{0}$. Then there is a natural smooth action of $G$ on $\tilde{M}$ so that the projection map $p: \tilde{M} \rightarrow M$ is equivariant. It follows that for $y \in \tilde{M}$ and $x=p(y) \in M$, we always have $G_{y} \subset G_{x}$. But we can say more. In fact, $G_{y}$ is normal in $G_{x}$ and $G_{x} / G_{y}$ is isomorphic to a subgroup of the fundamental group $\pi_{1}=\pi_{1}\left(M, x_{0}\right)$ of $M$. For if $g \in G_{x}$, then $p(g y)=g p(y)$ $=g x=x=p(y)$. Hence there is a unique $\alpha_{g} \in \pi_{1}$ such that $g y=y \alpha_{g}$. (We write the action of $\pi_{1}$ on $\tilde{M}$ on the right.) Then $\theta: g \mapsto \alpha_{g}$ is a homomorphism of $G_{x}$ into $\pi_{1}$ with $\operatorname{Ker} \theta=G_{y}$. In particular, as $G_{x}$ is compact and $\pi_{1}$ discrete, $G_{x} / G_{y}$ is finite. We can decide when $G_{x}=G_{y}$ as follows. Since $G$ is assumed to be connected, it is easily seen that the lifted action of $G$ on $\tilde{M}$ commutes with the action of $\pi_{1}$. Hence $\pi_{1}$ acts on the orbit space $\tilde{M} / G$ and the orbit space of this action can be identified with $M / G$. Now let $y \in \tilde{M}$ and $[y] \in \tilde{M} / G$ its orbit. It is easily seen that

$$
\left(\pi_{1}\right)_{[y]}=\theta\left(G_{x} / G_{y}\right) \text {. }
$$

Therefore $G_{y}=G_{x}$ iff $[y] \in \tilde{M} / G$ is free under the action of $\pi_{1}$, and this would always be the case if $G_{x}$ is connected. We will be interested in applying this in two extreme cases:

(1.1) If the principal isotropy subgroup type $(H)$ of $(G, M)$ is connected, then the action $(G, \tilde{M})$ also has $(H)$ as principal isotropy subgroup type.

(1.2) Since $G$ is connected, $F(G, \tilde{M})=p^{-1}(F(G, M))$. 
2. More preliminaries. Let $\left(Z_{2}, X\right)$ be a smooth involution where $X$ is a contractible $n$-manifold with boundary $\partial X$ such that $\left(Z_{2}, \partial X\right)$ is free. Then

(2.1) The fixed-point set $F\left(Z_{2}, X\right)$ is precisely a single point in the interior of $X$.

(2.2) The orbit space $X / Z_{2}$ is contractible.

(2.3) Let $D^{n} \subset$ Int $X$ be a disk around the fixed-point. Then the inclusion

$$
R P^{n-1}=\partial D^{n} / Z_{2} \rightarrow X-\operatorname{Int} D^{n} / Z_{2}
$$

is a homotopy equivalence.

(2.4) $\partial X / Z_{2}$ is a manifold having the same integral cohomology as $R P^{n-1}$. Moreover, if $\partial X$ is simply connected and $n \geqq 6$, then the action $\left(Z_{2}, \partial X\right)$ is equivalent to the standard antipodal involution $\left(Z_{2}, S^{n-1}\right)$, and hence $\partial X / Z_{2}$ is diffeomorphic to $R P^{n-1}$.

Proof. By P. A. Smith theory $F=F\left(Z_{2}, X\right)$ is acyclic over $Z_{2}$. Since $\left(Z_{2}, \partial X\right)$ is free, $F$ is a manifold without boundary. It follows that $F$ can only be a single point, say $x_{0} \in$ Int $X$. Let $D^{n} \subset$ Int $X$ be a disk around it. Consider the inclusion $\partial D^{n} \rightarrow X-$ Int $D^{n}$. From the exact triangle

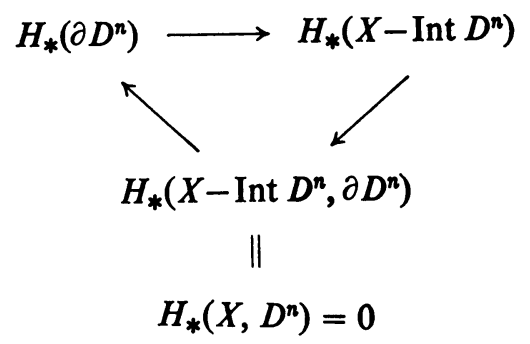

we see that $H_{*}\left(\partial D^{n}\right) \rightarrow H_{*}\left(X-\operatorname{Int} D^{n}\right)$ is an isomorphism. Also $X-\operatorname{Int} D^{n}$ is simply connected by the Van Kampen theorem. It follows (e.g. by a spectral sequence argument) that both

$$
H_{*}\left(\partial D^{n} / Z_{2}\right) \rightarrow H_{*}\left(X-\operatorname{Int} D^{n} / Z_{2}\right)
$$

and

$$
\pi_{1}\left(\partial D^{n} / Z_{2}\right) \rightarrow \pi_{1}\left(X-\operatorname{Int} D^{n} / Z_{2}\right)
$$

are isomorphisms. Therefore by Whitehead's theorem,

$$
R P^{n-1}=\partial D^{n} / Z_{2} \rightarrow X-\text { Int } D^{n} / Z_{2}
$$

is a homotopy equivalence.

Now consider the other end $\partial X$ of $X$-Int $D^{n}$. We have the exact triangle

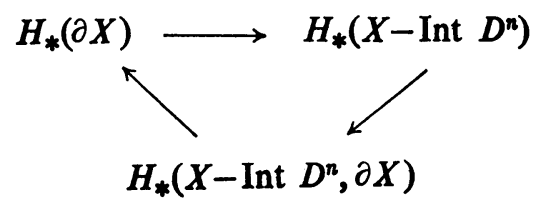


But Poincaré duality gives

$$
H_{*}\left(X-\text { Int } D^{n}, \partial X\right)=H^{*}\left(X-\operatorname{Int} D^{n}, \partial D^{n}\right)=H^{*}\left(X, D^{n}\right)=0 .
$$

Thus again $H_{*}(\partial X) \rightarrow H_{*}\left(X-\operatorname{Int} D^{n}\right)$ and hence $H_{*}\left(\partial X / Z_{2}\right) \rightarrow H_{*}\left(X-\operatorname{Int} D^{n} / Z_{2}\right)$ are isomorphisms. Thus $H_{*}\left(\partial X / Z_{2}\right)=H_{*}\left(R P^{n-1}\right)$. If $\partial X$ is simply connected, then

$$
\pi_{1}\left(\partial X / Z_{2}\right) \rightarrow \pi_{1}\left(X-\operatorname{Int} D^{n} / Z_{2}\right)
$$

would also be an isomorphism and hence the inclusion $\partial X / Z_{2} \rightarrow X-\operatorname{Int} D^{n} / Z_{2}$ is also a homotopy equivalence. But this means $X$-Int $D^{n} / Z_{2}$ is an $h$-cobordism between $\partial X / Z_{2}$ and $R P^{n-1}$. As $\mathrm{Wh}\left(Z_{2}\right)=0$, we conclude by the $s$-cobordism theorem that $X$-Int $D^{n} / Z_{2}$ is diffeomorphic to $R P^{n-1} \times I$ when $n \geqq 6$. This completes the proof of (2.1) through (2.4).

3. The Structure Theorem. Let $(G, M)$ be a smooth action of a compact connected Lie group on a compact connected manifold. Assume that all the orbits of the action are of uniform dimension. Then the connectedness of $M$ implies that all the identity components $G_{x}^{0}$ of the isotropy subgroups $G_{x}$ are conjugate, say of the same type $(H)$. Let $P=F(H, M)$ be the fixed-point set of $H, N=N(H, G)$ the normalizer of $H$ in $G$ and $K=N / H$ the quotient group. Then $P$ is a submanifold of $M$ invariant under $N$, so we have a natural action of $K$ on $P$. On the other hand, there is the natural right translation of $K$ on the homogeneous space $G / H$. Therefore we can form the space $G / H \times_{K} P$. This is a smooth manifold (since $K$ acts freely on $G / H$ ) on which $G$ acts naturally via left translations on $G / H$. It is easily seen that

$$
G / H \times{ }_{K} P \rightarrow M, \quad[g H, x] \mapsto g x
$$

is a diffeomorphism identifying $M$ with $G / H \times{ }_{K} P$ as $G$-manifolds. With this representation of $M$, for a point $m=g x$ with $g \in G, x \in P$, let $K_{x}$ be the isotropy subgroup of $x$ in the action $(K, P), \widetilde{K}_{x} \subset N$ the pull-back of $K_{x}$ under the projection $N \rightarrow K$. Then

$$
G_{g x}=g \tilde{K}_{x} g^{-1} \text {. }
$$

Thus $G_{g x}$ is of type $H$ iff $x \in P$ is free under $K$. For example if all orbits of $G$ on $M$ are actually of the same type $H$, then $K$ acts freely on $P$ and we get $M$ fibered over $P / K$ with fiber $G / H$ and group $K$, an observation due to A. Borel. The above situation, where each $K_{x}$ is permitted to be a finite group, is discussed in [2].

We shall need a slightly more general case than the above. Namely we assume $G$ has fixed-point set $F=F(G, M)$ but all other orbits are of the same dimension. Let $(H)$ be the principal orbit type. We assume that $G / H=S^{k}$ is a sphere of dimension $k \neq 1,3$. Let $U$ be an equivariant tubular neighborhood of $F$. Then $G$ acts on $M$-Int $U$ with uniform dimensional orbits and we can apply the above to represent $M$-Int $U$ by $G / H \times{ }_{K} P$, where $P=F(H, M-$ Int $U)$. Now we put $U$ back in. We have $U \rightarrow F$ a $D^{p}$ bundle over $F$ with group $G$ acting on $D^{p}$ via normal repre- 
sentation, where $p$ is the codimension of $F$ in $M$. Thus $\left(G, D^{p}\right)$ is a linear action with principal orbit type $G / H=S^{k}$. According to [4, Lemma 1, p. 425], if $k \neq 1,3$, then $p=k+1$ and the linear action of $G$ on the unit sphere $S^{p-1}=S^{k}$ is just the natural translation of $G$ on $G / H=S^{k}$. Now on $\partial U$, the diagram

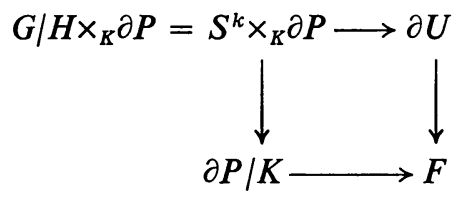

shows that the sphere bundle $\partial U \rightarrow F$ has $\partial P \rightarrow \partial P / K$ as its associated principal bundle. It follows that the disk bundle $U \rightarrow F$ can be identified with

$$
D^{k+1} \times_{K} \partial P \rightarrow \partial P / K
$$

and we may represent $M$ as

$$
M=S^{k} \times_{K} P \cup D^{k+1} \times_{K} \partial P,
$$

identified along $S^{k} \times_{K} \partial P$. Notice that from this representation, the orbit space can be given by

$$
M / G=P / K \cup[0,1] \times \partial P / K,
$$

with (1) $\times \partial P / K \subset[0,1] \times \partial P / K$ attached to $\partial P / K \subset P / K$. (For more details, see [8].)

We are ready to prove the structure theorem.

STRUCTURE THeOREM. Let $G$ be a compact connected Lie group acting smoothly on a homotopy real projective $m$-space $M$. Suppose the principal orbit of the action is a $k$-sphere $S^{k}$ with $k \neq 1,3$. Then

(i) There are precisely three types of orbits. Namely the sphere $S^{k}$, the real projective $k$-space $R P^{k}$, and fixed-points. Moreover there is exactly one orbit of type $R P^{k}$.

(ii) The fixed-point set $F=F(G, M)$ has the integral homology of $R P^{n}$, where $n=m-k-1$. If $\pi_{1}(F)=Z_{2}$ and $n \geqq 5$, then $F$ is actually diffeomorphic to $R P^{n}$.

(iii) Let $Y=M / G$ be the orbit space of the action $(G, M), y_{0} \in M / G$ the orbit of type $R P^{k}$ (see (i)) and $V$ a neighborhood of $y_{0}$ in $M / G$. Then $Y$ is a contractible space and $\left(Y, y_{0}\right)$ a relative manifold of dimension $n+1=m-k$. More precisely, $Y-$ Int $V$ is a smooth manifold with boundary $\partial(Y-$ Int $V)=F \cup R P^{n}$, the inclusion $R P^{n} \rightarrow Y$ -Int $V$ is a homotopy equivalence and $V$ is a cone over $R P^{n}$ with $y_{0}$ as vertex.

(iv) If $m \geqq 5$, then $M$ is diffeomorphic to $R P^{m}$.

Proof. (i), (ii), and (iii) are relatively simple. First of all, there must be a fixedpoint. For if not, then since $M$ has no rational cohomology, Theorem 4 of [4] applies to assert that the orbits of the action have uniform dimension. As mentioned above, we can write $M$ as

$$
M=G / H \times_{K} P=S^{k} \times_{K} P .
$$


Consider the homotopy exact sequence

$$
\cdots \rightarrow \pi_{k+1}(M) \rightarrow \pi_{k}(K) \rightarrow \pi_{k}\left(S^{k} \times P\right) \rightarrow \pi_{k}(M) \rightarrow \cdots .
$$

Since $K$ acts freely on $S^{k}$, it has rank 1 and so $\pi_{k}(K)$ is finite, for $k \neq 1,3$ (the identity component $K^{0}$ is either $S^{1}$ or $\left.S^{3}\right)$. As $\pi_{k}\left(S^{k} \times P\right)$ is infinite, we have $\pi_{k}(M) \neq 0$. This is impossible since $k<m$ and $M$ is a homotopy $R P^{m}$.

Thus we can apply the results of $\S 1$. Let $\tilde{M}$ be the universal covering of $M$ and $(G, \tilde{M})$ the lifted action. Since the principal orbit $G / H=S^{k}$ is simply connected, $H$ is connected, and (1.1) implies that $(G, \tilde{M})$ also has principal orbit $S^{k}$. Of course $\tilde{M}$ is a homotopy sphere and actions of this kind are completely understood by the work of W. C. Hsiang and W. Y. Hsiang [4]. For example there are only orbits of type $(H)$ and fixed-points. Thus the only possible orbit types of $(G, M)$ are $(H)$, $(G)$ and $\left(G_{x}\right)$ with $G_{x} / H=\pi_{1}(M)=Z_{2}$, i.e. the only possible orbits are spheres $S^{k}$, fixed-points, and projective spaces $R P^{k}$.

Let $X=\tilde{M} / G$ be the orbit space of $(G, \tilde{M})$. We know that $\pi_{1}(M)=Z_{2}$ acts on $X$ with orbit space $X / Z_{2}=M / G=Y$, and a point $[x]=y_{0} \in Y$ has orbit $R P^{k}$ iff $x \in X$ is a fixed-point of $Z_{2}$. Now by [4], $X$ is a contractible manifold of dimension $m-k=n+1$ with $\partial X=F(G, \tilde{M})$. By $(1.2), F(G, \tilde{M})=p^{-1}(F(G, M))$, hence $Z_{2}$ acts freely on $\partial X$. We can then apply (2.1) to conclude that $F\left(Z_{2}, X\right)$ is precisely a single point $x_{0} \in X$. Hence there is exactly one orbit of type $R P^{k}$.

Let $D^{n+1}$ be a disk around $x_{0}$. Then $V=D^{n+1} / Z_{2} \subset Y$ is a neighborhood of $y_{0}$ in $Y$ and $V$ is a cone over $R P^{n}=\partial D^{n+1} / Z_{2}$ with $y_{0}$ as vertex. By (2.2), $Y$ is a contractible space and $Y$-Int $V$ is a manifold with boundary $\partial(Y-$ Int $V)=\partial X / Z_{2}$ $\cup R P^{n}=F \cup R P^{n}$. Statements (ii) and (iii) now follow from (2.2) through (2.4).

Now we proceed to prove (iv), which is our main concern. Let $U$ be an equivariant tubular neighborhood of $F$. As we have seen above, we have

$$
M-\text { Int } U=S^{k} \times_{K} P, \quad U=D^{k+1} \times_{K} \partial P,
$$

and

$$
M=S^{k} \times_{K} P \cup D^{k+1} \times_{K} \partial P .
$$

We know the orbit of type $R P^{k}$ is determined by a point $x_{0} \in P$ such that $K_{x_{0}}=Z_{2}$, and this point projects, of course, to the point $y_{0} \in Y$ in the orbit space. Now by the slice theorem, the orbit of $x_{0}$ in $P$ has a tubular neighborhood $W$ in $P$ given by $W=K \times{ }_{z_{2}} D^{n+1}$. The singular orbit $R P^{k}$ has therefore a tubular neighborhood $\hat{W}$ in $M$ given by

$$
\hat{W}=S^{k} \times_{K}\left(K \times_{z_{2}} D^{n+1}\right)=S^{k} \times_{z_{2}} D^{n+1}
$$

and $\hat{W}$ projects to $\hat{W} / G=D^{n+1} / Z_{2}=V$, the cone neighborhood of $y_{0} \in Y$. Hence $Y$-Int $V$ is covered by

$$
M-\text { Int } \hat{W}=S^{k} \times_{K}(P-\text { Int } W) \cup D^{k+1} \times_{K} \partial P .
$$

Now consider the principal $K$-bundle

$$
\xi: P-\text { Int } W \rightarrow(P-\text { Int } W / K) \sim(Y-\text { Int } V) .
$$


Here " $"$ means of the same homotopy type, as $Y$-Int $V$ is obtained from $P$-Int $W / K$ by attaching a collar $[0,1] \times \partial P / K$. Now $\partial V=R P^{n} \subset Y-$ Int $V$ is a homotopy equivalence by (iii). Over $\partial V$, the bundle $\xi$ is given by

$$
\xi \mid \partial V: \partial W=K \times_{z_{2}} S^{n} \rightarrow S^{n} / Z_{2}=R P^{n}=\partial V .
$$

This means the bundle $\xi \mid \partial V$ has a $Z_{2}$-reduction. Since $\partial V \rightarrow Y-I n t V$ is a homotopy equivalence, there is a unique $Z_{2}$-reduction of $\xi$ which extends the reduction of $\xi \mid \partial V$. That is, there is a principal $Z_{2}$-bundle $Q \rightarrow Y$-Int $V$, with $Q$ a smooth manifold with boundary $\partial Q=S^{n} \cup B$, over $\partial V=R P^{n}$ and $F \subset Y-$ Int $V$ respectively, and an identification $K \times{ }_{z_{2}} Q=P$-Int $W$ compatible with the identification $K \times_{z_{2}} S^{n}=\partial W$. We can therefore represent $P$ as

$$
P=K \times_{z_{2}} Q \cup K \times_{z_{2}} D^{n+1}=K \times_{z_{2}} R,
$$

where $R=Q \cup D^{n+1}$ is obtained from $Q$ by adjoining a disk along $S^{n}$. Notice $R$ has a smooth action of $Z_{2}$ with exactly a single fixed-point. Now we can write $M$ as

$$
\begin{aligned}
M & =S^{k} \times_{K} P \cup D^{k+1} \times_{K} \partial P \\
& =S^{k} \times_{K}\left(K \times_{z_{2}} R\right) \cup D^{k+1} \times_{K}\left(K \times_{z_{2}} \partial R\right) \\
& =S^{k} \times_{z_{2}} R \cup D^{k+1} \times_{z_{2}} \partial R=\partial\left(D^{k+1} \times R\right) / Z_{2} .
\end{aligned}
$$

The diagram

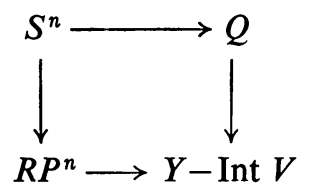

and the fact that $R P^{n} \subset Y-$ Int $V$ is a homotopy equivalence implies that $S^{n} \subset Q$ is a homotopy equivalence. Hence $R=Q \cup D^{n+1}$ is contractible and so is $D^{k+1} \times R$. Thus we have a smooth action of $Z_{2}$ on a contractible manifold, which is free on the boundary. Since $\partial\left(D^{k+1} \times R\right)$ is necessarily simply connected (e.g. by the Van Kampen theorem), we conclude from (2.4) that $M$ is diffeomorphic to $R P^{m}$ when $m \geqq 5$. This completes the proof of the Structure Theorem.

4. Main results. With the Structure Theorem we are now able to prove the following theorem:

THEOREM. If $M^{m}$ is an exotic $H R P^{m}(m \geqq 72)$, then

$$
N(M)<m^{2} / 8+1 \text {. }
$$

Proof. Suppose $M^{m}$ is a $H R P^{m}$ with $N(M) \geqq m^{2} / 8+1$. We proceed to show that $M^{m}$ is standard. Let $G$ be a compact connected Lie group of dimension $\geqq m^{2} / 8+1$ acting effectively and differentiably on $M$; let $H$ be a principal isotropy subgroup of the action. Let $\Sigma^{m}$ be the universal covering space of $M^{m}$. The action of $G$ on $M$ lifts to an effective differentiable action of a covering group $\tilde{G}$ of $G$ on $\Sigma^{m}$. Now $\operatorname{dim} \tilde{G}=\operatorname{dim} G$. 
Case A. $\operatorname{dim} G / H=m$.

In this case the action of $G$ on $M$, and hence, $\tilde{G}$ on $\Sigma^{m}$ is transitive. However, all transitive effective actions of compact connected Lie groups on spheres have been classified [11], [1], [13]. In all cases $\Sigma^{m}$ is diffeomorphic to $S^{m}$. We obtain the principal $Z_{2}$-bundle

$$
\begin{aligned}
S^{m} & =\tilde{G}_{1} / \tilde{H}_{1} \\
& \downarrow \\
\downarrow & \tilde{H}_{0} / \tilde{H}_{1} \\
M^{m} & =\tilde{G}_{1} / \tilde{H}_{0}
\end{aligned}
$$

where $\tilde{G}_{1}=S O(m+1), S U((m+1) / 2)$ or $S p((m+1) / 4)$ and $\tilde{H}_{1}=S O(m), S U((m-1) / 2)$ or $S p((m-3) / 4)$ (respectively) standardly imbedded. Now the free action of $\tilde{H}_{0} / \tilde{H}_{1}$ on $S^{m}$ is the restriction of the orthogonal action of $N\left(\tilde{H}_{1}, \tilde{G}_{1}\right) / \tilde{H}_{1}$ on $S^{m}$. It follows that $M^{m}$ is diffeomorphic to $R P^{m}$.

Case B. $\operatorname{dim} G / H=m-1$.

Now $\tilde{G}$ acts on $\Sigma^{m}$ with principal orbit also of codimension one. H. C. Wang has classified the groups acting effectively on spheres with principal orbit of codimension one [14], [6, p. 355]. It follows that $\tilde{G}$ is locally isomorphic to a subgroup of one of the following:

(i) $S O(t) \times S O(2), m=2 t-1$,

(ii) $U(t) \times U(2), m=4 t-1$,

(iii) $S p(t) \times S p(2), m=8 t-1$.

However in all cases,

$$
\operatorname{dim} G=\operatorname{dim} \tilde{G}<m^{2} / 8+1,
$$

which is a contradiction.

Case C. $\operatorname{dim} G / H \leqq m-2$.

Now

$$
\operatorname{dim} G \geqq m^{2} / 8+1 \geqq\left(\left(m^{2}+8\right) / 8(m-2)\right) \operatorname{dim} G / H
$$

Hence

$$
\operatorname{dim} G \geqq r \operatorname{dim} G / H
$$

where

$$
r=\left(m^{2}+8\right) / 8(m-2) .
$$

It follows from results of W. Y. Hsiang [7, Propositions 1 and 2] that there exists a simple connected normal subgroup $G_{1}$ of $G$ such that

$$
\operatorname{dim} G_{1} \geqq r\left[m\left(G_{1}\right)\right],
$$

and

$$
\operatorname{dim} G_{1}+\operatorname{dim} N\left(H_{1}, G_{1}\right) / H_{1} \geqq r \operatorname{dim} G_{1} / H_{1},
$$


where

(i) $m\left(G_{1}\right)=$ smallest positive codimension of proper subgroups of $G_{1}$,

(ii) $H_{1}=G_{1} \cap H$,

(iii) $N\left(H_{1}, G_{1}\right)=$ normalizer of $H_{1}$ in $G_{1}$.

Since $m$, and consequently $r$, are large it follows from (4.3) and a knowledge of the maximal dimensions of proper subgroups of the exceptional Lie groups [8] that $G_{1}$ is a classical Lie group. Therefore $G_{1}$ is locally isomorphic to $S O(n), S U(n)$, or $S p(n)$. We consider only the case where $G_{1}$ is locally isomorphic to $S O(n)$ as the other two cases are similar and less difficult.

Since $m(S O(n))=n-1$, it follows from (4.3) that

$$
n(n-1) / 2=\operatorname{dim} S O(n) \geqq\left(\left(m^{2}+8\right) / 8(m-2)\right)(n-1) .
$$

and

$$
n>m / 4
$$

after simplifying. Since we are assuming that $m \geqq 72$ it follows from (4.5) that

$$
(n-1)^{2} \geqq 4 m \text {. }
$$

Now $M$ has zero first rational Pontrjagin class and with (4.6) we may conclude that $H_{1}^{0}$, the identity component of $H_{1}$, is locally isomorphic to $S O(n-k), 1 \leqq k \leqq n$ [5, Theorem 2.1]. We show $k \leqq 4$. By the underlying hypothesis of Case C, $\operatorname{dim} G / H$ $\leqq m-2$. Hence

$$
m-2 \geqq \operatorname{dim} G_{1} / H_{1}=\operatorname{dim} S O(n)-\operatorname{dim} S O(n-k)
$$

and consequently

$$
m \geqq n k-k(k+1) / 2+2 .
$$

Combining (4.5) with (4.7) yields

$$
4 n>n k-k(k+1) / 2+2
$$

and since $n$ is large it follows that $k \leqq 4$. For $k=3,4$ we may employ (4.4) directly to obtain an easy contradiction. Consider then $k=2$. From (4.4) we obtain

$$
\operatorname{dim} S O(n)+\operatorname{dim} S O(2) \geqq\left(\left(m^{2}+8\right) / 8(m-2)\right) \operatorname{dim} G_{1} / H_{1}
$$

or

$$
n(n-1) / 2+1 \geqq\left(\left(m^{2}+8\right) / 8(m-2)\right)(2 n-3) .
$$

Now it follows from (4.5) and (4.7) that $m / 4<n \leqq(m+1) / 2$. Let

$$
f(n)=n(n-1) / 2+1-r(2 n-3)
$$

where, as we recall, $r=\left(m^{2}+8\right) / 8(m-2)$. We proceed to reach a contradiction to (4.9) by showing that $f(n)<0$ for $m / 4<n \leqq(m+1) / 2$. 
It is easily checked that $f((m+1) / 2)<0$ and $f(m / 4)<0$. Since $f(n)$ is concave upward, the contention follows.

We are left with $k=1$. The almost effective action of $S O(n)$ on $M$ may be lifted to an almost effective action of $S O(n)$ on $\tilde{M}$, the universal covering space of $M$. Since $\tilde{M}$ is a homotopy sphere and since $(n-1)^{2} \geqq 4 m$, it follows from a result of the Hsiang brothers [5, Theorem 3.1] that $S O(n)$ acts on $\tilde{M}$ with principal orbit $S^{n-1}$ and with a nonempty fixed-point set. From our considerations in the last section we know now that $S O(n)$ acts on $M$ with a fixed-point. Now the principal orbit of the action of $S O(n)$ on $M$ is either $S^{n-1}$ or $R P^{n-1}$. However, since $S O(n)$ acts linearly in a neighborhood of a fixed-point of $M$, it follows that the principal orbit is actually $S^{n-1}$. Finally the Structure Theorem applies and $M$ is diffeomorphic to $R P^{m}$.

EXAMPLE. We employ the Hirzebruch-Brieskorn construction of homotopy spheres to show that this theorem is best possible. Let $\tilde{M}^{8 k+1}$ be the intersection of the complex hypersurfaces

$$
Z_{1}^{2}+Z_{2}^{2}+\cdots+Z_{4 k+1}^{2}+Z_{4 k+2}^{2}=0
$$

and

$$
\left|Z_{1}\right|^{2}+\left|Z_{2}\right|^{2}+\cdots+\left|Z_{4 k+2}\right|^{2}=1
$$

in $C^{4 k+2}$. It is known that $\tilde{M}^{8 k+1}$ is diffeomorphic to the Kervaire sphere $\Sigma_{0}^{8 k+1}$ (see, for example, [12, pp. 55-56]). It is easily seen that a standard subgroup $O(4 k+1)$ in $U(4 k+2)$ leaves $\tilde{M}^{8 k+1}$ invariant in $C^{4 k+2}$. In addition there is an $S^{1}$ action on $\tilde{M}^{8 k+1}$ which commutes with the $O(4 k+1)$ action. Namely, if $w=e^{i \theta} \in S^{1}$,

$$
w\left(Z_{1}, Z_{2}, \ldots, Z_{4 k+1}, Z_{4 k+2}\right)=\left(e^{3 i \theta} Z_{1}, e^{3 i \theta} Z_{2}, \ldots, e^{3 i \theta} Z_{4 k+1}, e^{2 i \theta} Z_{4 k+2}\right) .
$$

We obtain an almost effective action of $O(4 k+1) \times S^{1}$ on $\tilde{M}$. Consider the involution $t=e^{i \pi}=-1$ in $S^{1}$. Clearly $t$ acts freely on $\tilde{M}$. Let $A$ be the group of order two generated by $t$ and let $M^{8 k+1}=\tilde{M}^{8 k+1} / A$. Now $M$ is a homotopy $R P^{8 k+1}$ and, since its universal covering space $\tilde{M}$ is an exotic sphere, $M$ is not diffeomorphic to $R P^{8 k+1}$. On the other hand, the almost effective action of $O(4 k+1) \times S^{1}$ on $\tilde{M}$ can be pushed down to an almost effective action on $M$. Hence if $m=8 k+1$,

$$
N(M) \geqq \operatorname{dim}\left[O(4 k+1) \times S^{1}\right]=8 k^{2}+2 k+1=m^{2} / 8+7 / 8 .
$$

REMARKS. 1. It is possible, by a somewhat more careful argument, to lower the bound in the preceding theorem to $m \geqq 59$.

2. The statements and proofs of the above results and of the example can be modified so as to apply to $Z_{3}$ actions on spheres, since the Whitehead group of $Z_{3}$ is zero. In particular, if $M^{m}$ is an exotic lens space arising from such an action, $N\left(M^{m}\right)<m^{2} / 8+1$. On the other hand, it follows from the main lemma in [10] that, for a standard simple lens space $L_{p}^{m}$, with $p \neq 2, N\left(L_{p}^{m}\right)=m^{2} / 4+m / 2+1 / 4$. 


\section{REFERENCES}

1. A. Borel, Le plan projectif des octaves et les sphères comme espaces homogènes, C. R. Acad. Sci. Paris 230 (1950), 1378-1380. MR 11, 640.

2. P. E. Conner, Orbits of uniform dimension, Michigan Math. J. 6 (1959), 25-32. MR 21 \#4996.

3. L. P. Eisenhart, Riemannian geometry, Princeton Univ. Press, Princeton, N. J., 1949. MR 11, 687.

4. W. C. Hsiang and W. Y. Hsiang, Classification of differentiable actions of $S^{n}, R^{n}$, and $D^{n}$, with $S^{k}$ as the principal orbit type, Ann. of Math. (2) 82 (1965), 421-433. MR 31 \#5922.

5. - Differentiable actions of compact connected classical groups. I, Amer. J. Math. 89 (1967), 705-786. MR 36 \#304.

6. W. Y. Hsiang, On the bound of the dimensions of the isometry groups of all possible riemannian metrics on an exotic sphere, Ann. of Math. (2) 85 (1967), 351-358. MR 35 \#4935.

7. - On the degree of symmetry and the structure of highly symmetric manifolds, University of California, Berkeley, Calif. (mimeo.).

8. K. Janich, Differenzierbare G-Mannigfaltigkeiten, Springer-Verlag, Berlin and New York, 1968. MR 37 \#4835.

9. J. Kister and L. N. Mann, Isotropy structure of compact Lie groups on complexes, Michigan Math. J. 9 (1962), 93-96. MR 24 \#A1967.

10. H. T. Ku, L. N. Mann, J. L. Sicks and J. C. Su, Degree of symmetry of a product manifold, Trans. Amer. Math. Soc. 146 (1969), 133-149. MR 40 \#3579.

11. D. Montgomery and H. Samelson, Transformation groups of spheres, Ann. of Math. (2) 44 (1943), 454-470. MR 5, 60.

12. P. S. Mostert, Proceedings of the conference on transformation groups, Springer-Verlag, New York, 1968. MR 39 \#4875.

13. J. Poncet, Groupes de Lie compacts de transformations de l'espaces euclidean et les sphères comme espaces homogènes, Comment. Math. Helv. 33 (1959), 109-120. MR 21 \#2708.

14. H. C. Wang, Compact transformation groups of $S^{n}$ with an $(n-1)$-dimensional orbit, Amer. J. Math. 82 (1960), 698-748. MR 29 \#1285.

University of Massachusetts, Amherst, Massachusetts 01002 